\title{
Near-tip Fields of Plane-strain Crack Growing in Compressible Elastic Perfectly Plastic Material
}

\author{
K. C. HWANG and X. F. LUO
}

Department of Engineering Mechanics, Tsinghua University, Beijing, PRC

\section{ABSTRACT}

For plane-strain crack growing in compressible elastic perfectly plastic material, the near-tip field has 5-sector structure. There were intense discussions about the difference of solutions. In this paper a correct formulation is given and the solutions for stresses, flow factor and plastic strains are given for all the near-tip fields.

\section{KEYWORDS}

Crack growth; compressible material; near-tip fields; elastic perfectly plastic medium; plane strain crack.

\section{INTRODUCTION}

The solution with 4-sector structure given independently by Slepyan, Gao and Rice for near-tip fields of plane-strain crack growing steadily in incompressible $\left(\boldsymbol{\nu}=\frac{1}{2}\right)$ elastic perfectly plastic material is now widely accepted. But there were discussions if compressibility of material is considered $\left(\nu<\frac{1}{2}\right)$. Rice et al.(1980) extended the 4-sector solution to the case of compressible material. Gao(1981) pointed out that the yield condition is violated in the unloading sector of the 4-sector solution, and gave a 5sector solution(Fig.1) with strain singularity ahead of the tip. Drugan et al.(1982) obtained another 5-sector solution without strain singularity ahead of the tip. In recent works, the present authors noticed that in the solution given by Drugan et al.(1982) the flow factor $\lambda$ occurs to be negative and hence the plastic flow law is violated in part of the "nonsingular plastic sector" C (after the terminology by Rice(1982)). With the formulation in terms of Airy's stress function, the present paper gives the correct 5-sector solution. The limiting process of the degeneration of the present solution to the solution for incompressible material as $\nu \rightarrow \frac{1}{2}$ is studied by Hwang and Luo(1988). The results obtained coincide with those obtained by Luo and Hwang(1988) within the framework developed by Rice(1982 and refined in the spirit of this paper. 


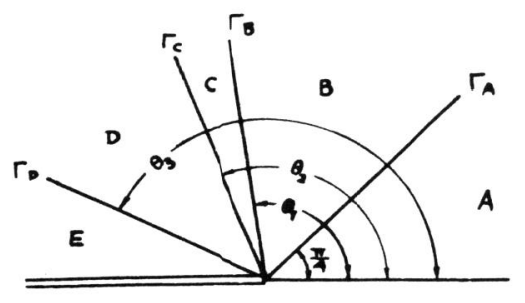

Fig.1. The 5-sector construction of near-tip field

BASIC EQUATIONS ( Gao and Hwang(1981a) )

Denote by $r, \theta$ the polar coordinates centered at crack tip. Take the leading $\phi(r, \theta, \nu)=r^{2} F(\theta, \nu)$

where $\nu$ is Poisson's ratio. Then we have

$$
\sigma_{r}=F^{\prime \prime}+2 F \quad \sigma_{0}-2 F \quad \sigma_{r e}=-F^{\prime}
$$

where a prime is used to denote derivative with respect $\theta$. Yield condition can be written as

$$
\frac{1}{4} F^{\prime 2}+F^{2}+\frac{1}{3} \epsilon^{2} \varphi^{2}=\tau^{2}
$$

where

$$
\epsilon=\frac{1}{2}-\nu \quad \zeta=-1.55_{33} / \epsilon
$$

Constitutive law for perfect plasticity is as follows

$$
\dot{\varepsilon}_{i j}=\frac{1+\nu}{E} \dot{\sigma}_{i j}-\frac{\nu}{E} \delta_{i j} \dot{q}_{k \mathrm{k}}+\lambda S_{i j} \quad(i, j, k=1,2,3)
$$

For quasi-static steady crack growth, we can assume speed of crack growth $a=1$ and then have for any quantity ( )

$$
(\dot{ })=-\left(\cos \theta \frac{\partial}{\partial r}-\sin \theta \frac{1}{r} \frac{\partial}{\partial \theta}\right)()
$$

Then $(2)$ can be rewritten in the asymptotic form $(r \rightarrow 0)$

$$
\dot{\varepsilon}_{i j}=\frac{1}{r} \sin \theta\left(\frac{1+\nu}{E} \sigma_{i j}^{\prime}-\frac{\nu}{E} \delta_{i j} \sigma_{k K}^{\prime}\right)+\lambda s_{i j} \quad(i, j, k=1,2,3)
$$

In plane-strain condition $\dot{\varepsilon}_{33}=0$, and from (4) it follows that

$$
\rho^{\prime}+\frac{2}{3} \frac{1}{\sin \theta} E r \lambda S=F^{\prime \prime \prime}+4 F^{\prime}
$$

The compatibility and rate of compatibility equations are, respectively, $\frac{1-\nu^{2}}{E} \Delta \Delta \phi+\frac{\partial^{2} \varepsilon_{1}^{p}}{\partial x_{2}^{1}}+\frac{\partial^{2} \varepsilon_{2 q}^{p}}{\partial x_{1}^{2}}-2 \frac{\partial^{2} \varepsilon_{1}^{p}}{\partial x_{1} \partial x_{2}}=0$

$\frac{1-\nu^{2}}{E} \Delta \Delta \frac{\partial \phi}{\partial x_{1}}-\frac{1}{2}\left(\nabla_{1} \nabla^{\prime}-\nabla_{2} \nabla^{2}\right) \phi \cdot\left(\nabla_{1} \nabla^{\prime}-\nabla_{2} \nabla^{2}\right) \lambda-2\left(\nabla_{1} \nabla^{2} \lambda\right)\left(\nabla_{2} \nabla^{\prime} \phi\right)$

$-\frac{1}{2} \lambda \Delta \Delta \phi-\left(\nabla_{\alpha} \lambda\right)\left(\nabla^{\alpha} \Delta \phi\right)-\frac{2}{3} \epsilon^{2} \Delta(\lambda \xi)-0 \quad(\alpha-1,2)$

The in-plane plastic strain components can be obtained by integration

$$
\begin{aligned}
& \varepsilon_{11}^{p}=\frac{1}{2} \int_{x_{1}}^{x_{1}} \lambda\left(\sigma_{11}-\sigma_{22}\right) d x_{1}+\epsilon^{2} \frac{1}{E}\left(\sigma_{11}+\sigma_{22}-\xi\right) \\
& \varepsilon_{22}^{p}=\frac{1}{2} \int_{x_{1}}^{x_{1}} \lambda\left(\sigma_{22}-\sigma_{11}\right) d x_{1}+\epsilon^{2} \frac{1}{E}\left(\sigma_{11}+\sigma_{22}-\xi\right)
\end{aligned}
$$

$$
\varepsilon_{12}^{p}=\int_{x_{1}}^{x_{A}} \lambda \sigma_{12} d x_{1}
$$

The bordering line $r$ between the ahead plastic loading sector and the rear lastic unloading sector ( see $r_{c}$ in Fig.1) is called " the unloading elastic unloading sector ( see $r$ coundary for the case boundary ". We restate the the of elastic perfect pows:

Theorem: If on an unloading boundary $\Gamma$ we have at the side of the plastic sector (denoted by $\Gamma(p)$ )

$$
\epsilon s \neq 0 \text { or } \quad \sigma_{m}-\sigma_{\infty} \neq 0 \text { at } \Gamma(p)
$$

then

$$
\left.\lambda\right|_{r(p)}=0
$$

th should be noted that the conclusion (10) is, indeed, nontrivial. Actually, for the case of incompressible material, (10) is not true at the unloading boundary $r$ between the plastic centered fan sector and the elastic unloading sector, and we have a discontinuity jump of $\lambda$ across $r$. The reason is that both equalities in (9) then hold. It is not intuitively evident why $\lambda$ should be continuous across $r$ for the case of compressible material. This explains why the condition (10) ls liable to be disregarded. we refer the mathematical proof to Gao and Hwang(1981a,1983a).

Besides, symmetry about the crack line requires

$$
F^{\prime}=0 \quad \text { as } \quad \theta=0
$$

and traction-free conditions of crack surface require

$$
\begin{aligned}
& F=F^{\prime}=0 \quad \text { as } \quad \theta=\pi \\
& \text { DISCONTINUITY CONDITIONS }
\end{aligned}
$$

Gao and Hwang(1981b,1983a,b) proved that the rate of compatibility equation (7) can be rewritten as

$$
A_{1111} \frac{\partial^{A} \dot{\phi}}{\partial x_{1}^{4}}+A_{1112} \frac{\partial^{4} \dot{\phi}}{\partial x_{1}^{3} \partial x_{2}}+A_{1122} \frac{\partial \dot{\phi}}{\partial x_{1}^{2} \partial x_{2}^{3}}+\cdots=0
$$

where

$$
A_{1111}=\frac{16 \epsilon^{2}}{9} s^{2}\left(1-\nu^{2}\right)+\left(\sigma_{2 z}-\sigma_{11}+\frac{4}{3} \epsilon^{2} s\right)^{2}
$$

The characteristic equation of (13) is

$$
A_{1 I I !}=0
$$

which can be satisfied only when

$$
\left\{\begin{array}{l}
\epsilon \zeta=0 \\
\sigma_{11}=\sigma_{22}
\end{array}\right.
$$

(14b) gives the orientation of the characteristic line. Gao and Hwang

(1981b,1983a,b) also proved that only the shear strain may suffer discontinuity. Moreover, the line of discontinuity is certainly characteristic line, where both of (14) are satisfied.

The reasoning of Gao and Hwang leading to (14) is correct. Its direct consequence for compressible material $(\epsilon \neq 0)$ is 


$$
\left\{\begin{array}{l}
\zeta=0 \\
\sigma_{11}=\sigma_{23}
\end{array}\right.
$$

(15a,b) are the results obtained later by Drugan and Rice(1984,eq.(4.16b)). Unfortunately, Gao and Hwang drew an excessive conclusion that the two conditions in (15) cannot be satisfied simutaneously along a curve $\Gamma$, and hence, the basic equation is elliptic. In fact, if $\lambda \sim 0((\ln r) / r)$ in a zone ( for instance, singular plastic sector in this paper), then from (5) we see that in this whole zone

$$
\zeta=0
$$

Whe characteristic line where $(14 \mathrm{~b})$ is satisfied.

The contiguity conditions given by Gao and Hwang(1981a,1983a) for bordering line $r$ between neighboring sectors are:

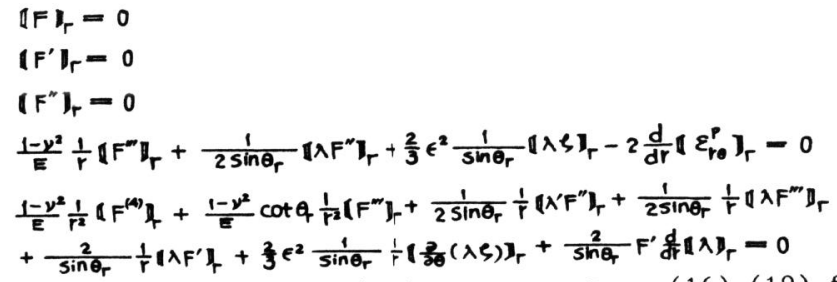

where where $y_{r}$ is used the difference of compatibility equation (7) at both sides of $r$. It should be emphasized that in deriving (16)-(20) we have assumed a possible singularity of curvature of $r$ at crack-tip which has the order less than $(\ln r)^{-1 / r}$ as $r \rightarrow 0$. Besides, these contiguity conditions are correct since its derivation $\overrightarrow{i s}$. Bet all influenced by the abcve-mentioned excessive conclusion drawn

by Gao and Hwang.

ASYMPTOTIC SOLUTION OF NEAR-TIP FIELDS

The 5-sector structure is shown in Fig.1, where A---constant stress sector, The 5-sector stactic sector, $\mathrm{C}$ and E--nonsingular plastic sectors, and $\mathrm{D}-$ B

$\underline{\text { Sector A }}$

$F=\frac{\tau_{0}}{2}(b+\cos 2 \theta)$ $\lambda=O(1)$

$\sigma_{11}=\tau_{0}(b-1) \quad \sigma_{22}=\tau_{0}(b+1)$

$\sigma_{12}=0$

From (1) and (8) we obtain, respectively,

$$
\zeta=0
$$

$\varepsilon_{\alpha p}^{p}=0(1)$

$(\alpha, \beta=1,2)$

Sector

$$
F=\frac{\tau_{0}}{2}\left\{b-2\left(\theta-\frac{\pi}{4}\right)\right\} \quad \lambda=A \frac{1}{r} \ln r+O\left(\frac{1}{p}\right)
$$

follows from the yield condition (1)

At the already satisfied. From the $4 \mathrm{th}$ and 5 th contiguity conditions, we have

$$
\begin{aligned}
& \left.\mid \varepsilon_{r o}^{p}\right)_{r_{A}}=\frac{1}{2}(3+4 \epsilon) \frac{\tau_{0}}{E} \ln \frac{\bar{R}}{r} \\
& \left.\lambda=-\frac{\sqrt{2}}{r}\left(\frac{3}{x}+\epsilon\right) \frac{\ln r}{r}+\alpha \frac{1}{r}\right)
\end{aligned}
$$

Plastic strain components in Sector B follow from (8)

$$
\begin{aligned}
& \varepsilon_{11}^{p}=-\varepsilon_{22}^{p}--\frac{2 \sqrt{2}}{E}\left(\frac{3}{4}+\epsilon\right) \sin \theta \ln \frac{\bar{\beta}}{p}+O(1) \\
& \varepsilon_{12}^{p}=\frac{\sqrt{2}}{E}\left(\frac{3}{4}+\epsilon\right)\left\{\cos \frac{\pi}{4}\left(\ln \frac{\tan (\theta / 2)}{\tan (\pi / 8)}+2 \cos \theta\right)-1\right\} \ln \frac{\bar{B}}{\gamma}+O(1)
\end{aligned}
$$

$\underline{\text { Sector C }}$

$$
\lambda=O\left(\frac{1}{T}\right) \quad s \rightarrow 0
$$

Making a consistent choice of sign, we obtain from (1)

$$
s--\frac{\pi}{\epsilon}\left(\tau_{0}^{2}-\frac{1}{4} F^{\prime 2}-F^{\prime 2}\right)^{1 / 2}
$$

Then from (5) and (7), we have

$$
\begin{aligned}
& \lambda=\frac{3}{2 E r} \frac{\sin \theta}{3}\left(F^{\prime \prime \prime}+4 F^{\prime}\right)\left(1+\frac{3}{4 E^{2} S^{\prime}} F^{\prime \prime}\right) \\
& \frac{1}{E}\left(\frac{3}{4}+\epsilon+\frac{3}{2 S} F^{\prime \prime}+\frac{9}{16 E^{2}} \frac{F^{\prime 2}}{3^{2}}\right)\left(F^{\prime \prime \prime}+4 F^{\prime}\right)=C_{1} \cot \theta+C_{2}
\end{aligned}
$$

where $C_{1}, C_{2}$ are integration constants. It can be proved that the plastic whe jump across $r_{B}$. From the 4 th and 5 th contiguity conditions (19) and (20), it follows, respectively,

\section{$C_{1}=-\frac{\sqrt{2}}{2} \frac{\tau_{0}}{E}(3+4 \epsilon) \sin \theta_{1}$ \\ $C_{2}=-\frac{\sqrt{2}}{2} \frac{\tau_{6}}{E}(3+4 \epsilon)\left(\sqrt{2}-\cos \theta_{1}\right)$}

$\zeta$ and $\lambda$ in Sector $C$ can be obtained by integrating (32), (33) and (34). F 5 , 5 an ang since starting values are taken from the power series expansion at $\theta_{1}$ obtained from (32), (33) and (34):

$F=\frac{\tau_{0}}{2}\left\{b-2\left(\theta_{1}-\frac{\pi}{4}\right)\right\}-\tau_{0}\left(\theta-\theta_{1}\right)+\frac{(3+4 \epsilon)^{2}}{240 \epsilon^{2} \sin ^{2} \theta_{1}} \tau_{0}\left(\theta-\theta_{1}\right)^{5}+\cdots$

$\zeta=-\frac{(3+4 \epsilon) \tau_{0}}{4 \sqrt{2} \epsilon^{2} \sin \theta_{1}}\left(\theta-\theta_{1}\right)^{2}+\cdots$

The integration of (34) for $F$ is performed from $\theta_{1}+0.1^{\circ}$ in increment of $0.0005^{\circ}$, and $\xi$ and $\lambda$ can be calculated from (32) and (33). The angle $\theta_{2}$ where $\lambda=0$ corresponds to the unloading bordering line $r_{c}$.

\section{Sector D}

In the elastic unloading sector, $\lambda=0$, and

$$
\varepsilon_{\alpha \beta}^{p}=\varepsilon_{\alpha \beta}^{p}\left(x_{2}\right) \quad(\alpha, \beta=1,2)
$$

Assume

AFR-1-Ne 
$\frac{\mathrm{d}^{2} \varepsilon_{1}^{p}}{d x_{2}^{\frac{1}{2}}}=\frac{D_{0}}{x_{2}^{2}}$

Then the solution of equation (6) is

$F=\frac{E}{1-\nu^{2}}\left\{D_{1}+D_{2} \theta+D_{3} \cos 2 \theta+D_{4} \sin 2 \theta+F^{*}\right\}$

where

$F^{*}=-\frac{D_{e}}{4}\left\{(\cos 2 \theta-1) \ln \sin \theta+\left(\theta+\frac{1}{2} \cot \theta\right) \sin 2 \theta\right\}$

The contiguity conditions (16)-(20) at $\Gamma_{c}$ lead to

$D_{1}+D_{2} \theta_{2}+D_{3} \cos 2 \theta_{2}+D_{4} \sin 2 \theta_{2}+F^{\prime \prime}\left(\theta_{2}\right)=\frac{1-\nu^{2}}{E} F_{c}\left(\theta_{2}\right)$

$D_{2}-2 D_{3} \sin 2 \theta_{2}+2 D_{4} \cos 2 \theta_{2}+F^{* \prime}\left(\theta_{2}\right)-\frac{1-\nu^{2}}{E} F_{c}^{\prime}\left(\theta_{2}\right)$

$-4 D_{3} \cos 2 \theta_{2}-4 D_{4} \sin 2 \theta_{2}+F^{* \prime \prime}\left(\theta_{1}\right)=\frac{1-\nu^{2}}{E} F_{c}^{\prime \prime}\left(\theta_{2}\right)$

$D_{2}=\frac{1}{4} C_{2}$

$D_{0}=C_{1}$

where $F_{C}, F_{c}^{\prime}, F_{c}^{\prime \prime}$ denote values in Sector $C$. The above equations can be used for obtaining $D_{1}, D_{2}, D_{3}, D_{4}$ and $D_{0}$ for given $\theta_{1}$ and $\theta_{2}$. From (5) we get

$S=F^{\prime \prime}+4 F+G$

where the constant $G$ can be obtained from the continuity of $\rho$ across $r_{c}$.

The plastic strain components in Sector D are

$$
\begin{aligned}
& \varepsilon_{11}^{p}=-\varepsilon_{22}^{p}=-\frac{\sqrt{2}}{2}(3+4 \epsilon) \frac{1}{E} \sin \theta_{1} \ln \frac{A \sin \theta_{1}}{x_{2}}+O(1) \\
& \varepsilon_{12}^{p}=\frac{\sqrt{2}}{4}(3+4 \epsilon) \frac{1}{E}\left\{\cos \frac{\pi}{4}\left(\ln \frac{\tan \left(\theta_{1} / 2\right)}{\tan (\pi / \theta)}+2 \cos \theta_{1}\right)-1\right\} \ln \frac{A \sin \theta_{1}}{x_{2}}+O(1)
\end{aligned}
$$

\section{Sector E}

$$
\lambda=O\left(\frac{1}{r}\right) \quad s \neq 0
$$

The governing equations for $\boldsymbol{\varphi}, \lambda$ and $\boldsymbol{F}$ in Sector $E$ remain to be (32), (33) and (34). It follows from the 4 th and 5 th contiguity conditions that the constants $C_{\text {, and }} C_{2}$ for sector $E$ remain the same as for Sector $C$. The

initial values necessary for starting the integration of (34) are offered by the first 3 contiguity conditions at $\Gamma_{\mathbf{p}}$.

Boundedness of each term in (5) as $\theta \rightarrow \pi$ implies

$$
e \rightarrow 0 \quad \text { as } \theta \rightarrow \pi
$$

Hence the integration of (34) cannot be performed directly to the free crack surface $(\theta=\pi)$. It is proved by Hwang and Luo(1988) that the condition $F^{\prime}=0$ in (12) can be replaced by

$$
\widetilde{F}^{2}=-\widetilde{F}^{\prime} \widetilde{F}^{\prime \prime} \eta+2\left(\widetilde{F}^{2}+\frac{1}{3} \epsilon^{2} \widetilde{S}^{\prime}\right) \eta^{2}
$$

where " $\sim$ " denotes the value taken at $\theta=\pi-\eta\left(\right.$ say, $\left.\eta=0.1^{\bullet}\right)$.

NUMERICAL RESULTS

For the asymptotic solution, we have to solve the differential equation (34) For the asymptotic solution, we have to solve the differential equation (34) $\Gamma_{B}, \Gamma_{c}, \Gamma_{0}\left(\right.$ Fig.1). It costitutes a one-parameter (i.e. $\left.\theta_{1}\right)$ shooting $\Gamma_{B}, \Gamma_{c}, \Gamma_{D}$ (Fig.1). It costitutes a one-parameter (i.e. $\left.\theta_{4}\right)$ shooting
. Assume a value of $\theta_{1}$;

$(35)$

3. Calculate from (36) the initial values necessary for starting the integration of (34) from $\theta=\theta_{1}+0.1^{\circ}$ (take, for convenience, for the time being $b=0$ );

4. Integrate (34), calculate pointwise values of $\lambda$, determine the angle for $r_{c}$ from the requirement (10);

5. Calculate from (39), (40) the constants $D_{1}, D_{2}, D_{3}, D_{4}, D_{0}$ and $G$ for ector D;

6. Determine from (1) the angle $\theta_{3}$ for the reloading plastic boundary $\Gamma_{D}$ 7. Calculate from the first 3 contiguity conditions the initial values at $\Gamma_{0}$ necessary for starting the integration of (34);

8. Integrate (34) from $\Gamma_{0}$ to $\theta=\pi-\eta$ (say, $\eta=0.1^{\bullet}$ ), and examine whether 8. Integrate (34) is satisfied. If it is not, then repeat the above set of the condtion (44) is satisfied within prescribed accuracy,

9. Determine condition $F(\pi)=0$ (see (12)).

In the solution by Drugan et al.(1982) the flow factor $\lambda$ turns out to be inctic flow law is violated in a portion of Sector C. negative, hence the plastict The reason for this ", replaced it by a (10) inplied by the un (33) ( (33) $\theta_{1}$ and $\theta_{2}$ shooting problenting solution for acceptable, since it does notrain singularity ahead of the crack-tip.

The full near-tip fields for stresses, strains and flow factor can be obtained. obtained. Here only angles obtained by present results sho wing by the present authors.

Table 1. Comparison of $\theta_{1}, \theta_{2}$ and $\theta_{3}$ between the results of present paper and those by Drugan et al. (1982)

\begin{tabular}{lccc}
\hline & $\theta_{1}$ & $\theta_{2}$ & $\theta_{3}$ \\
\hline Present results & $110.09^{\circ}$ & $118.20^{\circ}$ & $160.42^{\circ}$ \\
Drugan et al.(1982) & $110.26^{\circ}$ & $123.13^{\circ}$ & $160.38^{\circ}$ \\
\hline
\end{tabular}

REFERENCES

Drugan, W.J. and J.R. Rice(1984). Restrictions on quasi-statically moving surface of strong discontinuity in elastic-plastic solids. In: Mechanics of Material behavior, The Doniel C. Drucker Anniversablishers.

and R. . Shield, plane strain tensile crack in elastic-ideally-plastic solids. J. Mech. Phys. Solids, $30,447-473$.

Phys. Solids, $\frac{30}{\text {, }}$, 
field of a growing crack. Proceedings of ASTM Second International Symposium on Elastic-Plastic Fracture Mechanics, Philadelghia, 6-10, October, 1981 .

K.C. Hwang(1981a). Elastic-plastic fields in steady crack growth. In: Three-Dimensional Constitutive Relations and Ductile Fracture (S. Nemat-Nasser, ed.),pp.417-473. North-Holland Pub. Co. for perfectly Gao, Y.C. and K.C. Hwang(1981b). The plane strain problem for perfectly elastic-plastic medium. Acta Mechanica Sinica, Special Issue (1981)

120. for elastic perfectly-plastic medium. Int. J. of Engng. Sci., 21, 765-780. Gao, Y.C. and K.C. Hwang(1983b). The discontinuity in quasi-static plastic fields. Proc. of ICF Int. Symp. on Fracture Mech. (BeI Ing, 1983), pp. 24-30. Hwang, K.C. and X.F. Luo(1988). Near-tip fields for crackse in elastic-perfectly-plastic compressible matear Fracture Mechanics,

IUTAM Symposium on Recent Advances in Nonlin.

Caltech, Pasadena, CA,U.S.A., March 14-16,1988. Luo, X.F. and K.C. Hwang(1988). Correct formulation aterials, field problem for crack growing in compressibe

Rice, J.R., W.J. Drugan and T.-L. Sham(1980) 700,pp.189-219.

growing crack. Fracture Mechanics, As Rice, J.R.(1982). Elastic-plastic crack growth. In: Mechanics of Soll, ed.) Rodney Hill 60th Anniversary Volume. 